\title{
Genetics of the apolipoprotein-E isoprotein system in man
}

\author{
ALASTAIR M CUMMING AND FORBES W ROBERTSON \\ From the Department of Genetics, University of Aberdeen, Aberdeen.
}

SUMmARY The inheritance of the apoprotein-E isoprotein polymorphism in man has been studied by pedigree analysis. Alternative genotypes have been identified by the use of isoelectric focusing and two dimensional electrophoresis. A single locus three allele model can account for the inherited differences. In addition to the major bands there are also minor bands which may differ in charge or molecular weight or both, probably owing to post-translational modification. Their relative intensity has been compared in alternative categories of patient liable to raised serum lipoprotein concentration and only in hyperthyroid cases is there clear evidence of relative increase of posttranslational activity which is reduced after restoration of the euthyroid state.

Apolipoprotein-E or arginine rich peptide was first shown to be a major constituent of very low density serum lipoprotein (VLDL) by Shore and Shore. ${ }^{1}$ Separation of the apoprotein on SDS PAGE showed a wide band with an approximate molecular weight of $33000 .^{2}$ The possible importance of this apoprotein in lipoprotein metabolism was inferred from the increased levels of the apoprotein in the VLDL of patients with type III hyperlipoproteinaemia. ${ }^{3}$ Using the technique of isoelectric focusing, Utermann et al $^{4}$ showed that a number of isoproteins (Apo- $E_{0}, E_{1}, E_{2}, E_{3}$, and $E_{4}$ ) were present in the normal population, but in type III hyperlipoproteinaemia Apo- $E_{3}$ and $E_{4}$ were absent and this was confirmed in further studies. ${ }^{4}$ Family studies of first degree relatives of type III probands suggested that absence of these isoproteins in type III hyperlipoproteinaemia was inherited simply. Two autosomal co-dominant alleles, $\mathrm{E}^{\mathrm{n}}$ and $\mathrm{E}^{\mathrm{d}}$, were proposed for the control of the ratios of Apo- $E_{3} /$ Apo- $E_{2}$ in heterozygotes and homozygotes. An additional locus was postulated to account for the presence or absence of the $E_{4}$. However, this model failed to account for the absence of the $E_{4}$ isoprotein in type III hyperlipoproteinaemia and a more recent publication ${ }^{5}$ favoured two closely linked loci with linkage disequilibrium to explain the isoprotein patterns found in the various pedigrees.

Zannis and Breslow, ${ }^{6}$ using two dimensional electrophoresis, showed that apoprotein-E isoproteins differed not only in isoelectric point but also in molecular weight. Five major band types Received for publication 24 March 1982. were demonstrated and the presence of minor bands was ascribed to post-translational modification of the major bands. In this system, type III hyperlipoproteinaemia could be clearly distinguished by isoelectric focusing alone. More recently, the same workers, on the evidence from family studies, showed that a single locus three allele model could explain the inheritance of the different isoprotein patterns.

In the present study, to achieve a better understanding of the inheritance of the different isoprotein patterns, we have investigated 12 families using the technique of isoelectric focusing and two dimensional electrophoresis. Our findings are consistent with the single locus three allele model, but we also draw attention to variation in the relative band intensity within genotypes and suggest that such variation may depend on thyroid status.

\section{Methods}

Twelve families of different size were chosen for study. In two of them the proband had type III hyperlipoproteinaemia. Seven families were selected on the evidence of our earlier population study ${ }^{7}$ which had indicated a number of families which included subjects with low LDL (low density lipoprotein) values. The rest of the families were selected because of presumed heterozygosity with respect to the presence of either Apo- $\mathrm{E}_{3}$ or Apo- $\mathrm{E}_{4}$ bands among related persons.

Blood was obtained by venepuncture from suitable family members after a 12 to 14 hour fast. 
VLDL was isolated at $\mathrm{d} 1.007 \mathrm{~g} / \mathrm{ml}$ by overnight ultracentrifugation in a $10 \times 10$ rotor, using an MSE Prepspin (MSE, Crawley, UK). A second overnight spin at the same density was required to rid the sample of contaminating plasma proteins. Lipids were extracted from a suitable aliquot of VLDL with acetone:ether at $-20^{\circ} \mathrm{C}$ overnight, rewashed in the same solvent mixture for 2 hours, and finally washed for 1 hour in ether. The pelleted residues were dried down over nitrogen and dissolved in $200 \mu 18 \mathrm{~mol} / \mathrm{l}$ urea containing Tris $/ \mathrm{HCl}(10$ $\mathrm{mmol} / \mathrm{l}) \mathrm{pH} 8.6$ and dithiothreitol $(10 \mathrm{mmol} / \mathrm{l})$ with occasional mixing. The solution was allowed to stand at $4^{\circ} \mathrm{C}$ for 3 hours before isoelectric focusing. Urea solutions were prepared immediately before use or frozen until required (Urea Analar BDH, UK).

Gels were prepared and focused in $0.45 \times 10 \mathrm{~cm}$ constant bore tube, essentially following the method of Warnick et al. ${ }^{8} 1.2 \mathrm{ml}$ of gel solution gave a separating gel length of $0.75 \mathrm{~cm}$. Gels were focused for 16 hours at $250 \mathrm{~V}$ and protein stained for 6 days in Coomassie Blue G $250^{10} .{ }^{9}$ Destaining was for 3 days in $5 \%$ acetic acid before densitometric scanning on a Chromoscan Mark II (Joyce Loebl). The areas under the peaks were traced on paper, cut out, and weighed to provide estimates of relative band concentration.

The isoelectric focusing step in two dimensional electrophoresis was carried out as described above but in $0.25 \times 12 \mathrm{~cm}$ constant bore glass tubes (effective gel length $10 \mathrm{~cm}$ ). The VLDL apoprotein residue was taken up in $90 \mu \mathrm{l}$ denaturing buffer. SDS PAGE electrophoresis in the second dimension was performed on a Bio-Rad Vertical Slab Gel Apparatus using O'Farrell's method, ${ }^{10}$ as modified by Zannis and Breslow. ${ }^{6}$ Separating gel dimensions were $9.5 \times 14 \mathrm{~cm}$ and $0.75 \mathrm{~mm}$ thick. Fifteen minutes equilibration time was allowed between the two gel systems. After electrophoresis the gels were stained in $0.25 \%$ Coomassie Blue R in $50 \%$ ethanol: $10 \%$ acetic acid for 1 hour, destained in the same solvent mixture for 1 to 2 hours, and finally washed in two to three changes of water until a colourless background was achieved. Before drying on a Bio-Rad Gel Dryer, the gel was soaked for several hours in $4 \%$ glycerol.

\section{Results}

BAND PATTERNS

Visual inspection and densitometric scan of the apoprotein-E isoproteins obtained in family studies suggested that the patterns could be classified into two categories with either a single strongly stained major band in only one of three alternative isoelectric positions $(2,3,4$,$) or apparently major band 8$ in two of the positions $(2 / 3,3 / 4,4 / 2)$. Examples of the six band patterns are illustrated in fig 1: (a) (c), and (e) illustrate the presence of a single major

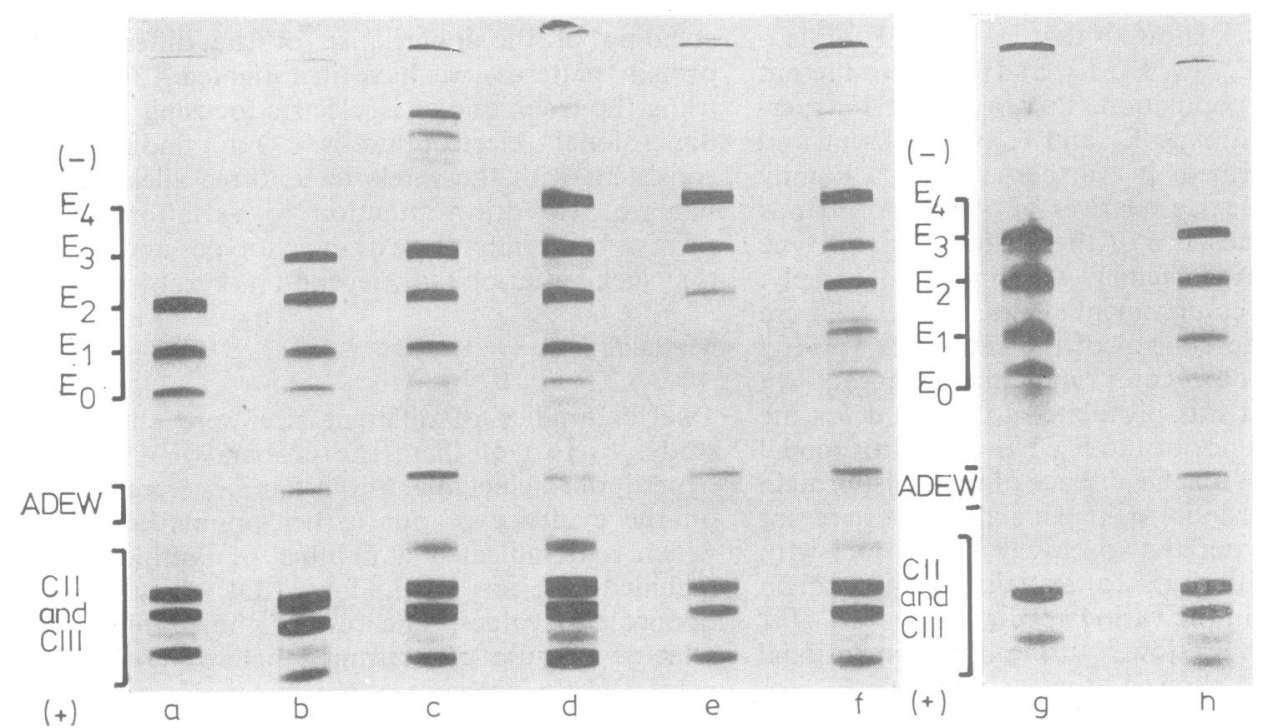

FIG 1 Isoelectric focusing patterns of Apo VLDL in six major genotypes: (a) $E_{\mathrm{II}} E_{\mathrm{II}}$; (b) $E_{\mathrm{II}} E_{\mathrm{III}}$; (c) $E_{\mathrm{III}} E_{\mathrm{III}}$; (d) $E_{\mathrm{III}} E_{\mathrm{IV}} ;(e) E_{\mathrm{IV}} E_{\mathrm{IV}} ;(f) E_{\mathrm{II}} E_{\mathrm{IV}} .(g)$ and $(h)$ show the Apo VLDL patterns of a hyperthyroid subject of genotype $E_{111} E_{11}$ in the affected and euthyroid state respectively. 
band, and (b), (d), and (f) the alternative combinations of two bands. In addition to these bands, used in the designation of genotypes, additional minor bands are present in the more anodic direction.

Although about $80 \%$ of the gels could be unambiguously typed from the isoelectric focusing sequence, the remainder were not so easily assigned, because of uncertainty as to the presence of one or two major bands. We therefore resorted to two dimensional electrophoresis. The complex two dimensional patterns are shown in fig 2. Single or double arrows indicate the presence of one or two major bands respectively. The letters attached to the two dimensional patterns correspond to those used for the particular unidimensional runs shown in fig 1 .

Three electrophoretograms, (a), (c), and (e), show a single major isoprotein, with minor isoproteins differing in charge or apparent molecular weight or both. The first 'minor' band seen in isoelectric focusing is resolved into two spots with the two dimensional procedure. One of these isoproteins has the same, or very similar, apparent molecular weight as the major isoprotein, while the

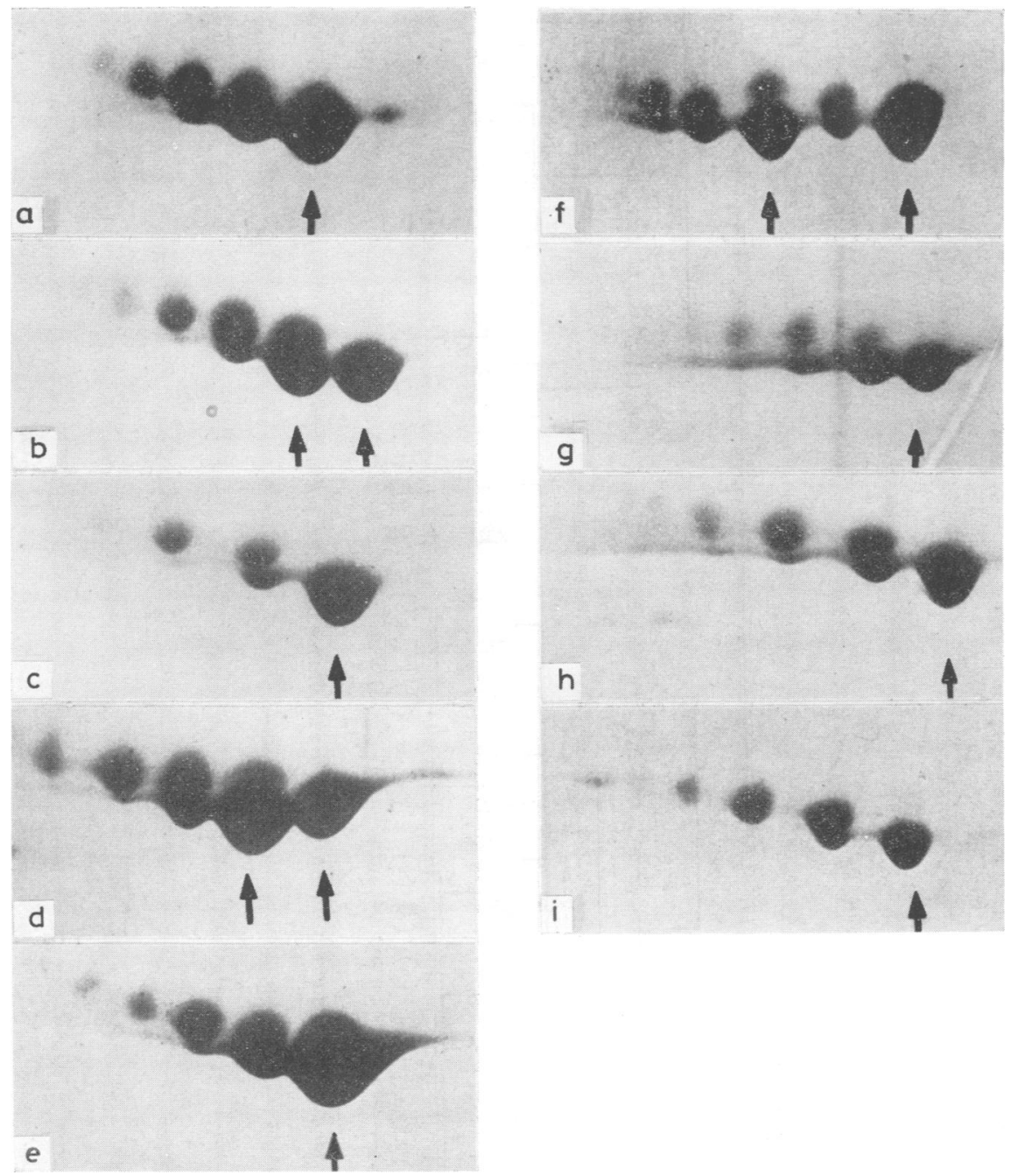

FIG 2 Two dimensional gel electrophoresis patterns of VLDL Apo-E showing the major Apo-E isoproteins. An arrow indicates a major band. The lettering of the genotypes $(a-h)$ corresponds to that in fig $1:(a) E_{\mathrm{II}} E_{\mathrm{II}} ;(b) E_{\mathrm{II}} E_{\mathrm{III}}$; (c) $E_{\mathrm{III}} E_{\mathrm{III}} ;(d) E_{\mathrm{III}} E_{\mathrm{IV}} ;(e) E_{\mathrm{IV}} E_{\mathrm{IV}} ;(f) E_{\mathrm{II}} E_{\mathrm{IV}} ;(g) E_{\mathrm{III}} E_{\mathrm{III}}$ hyperthyroid subject; (h) $E_{\mathrm{III}} E_{\mathrm{III}}$ same subject in euthyroid state; (i) $E_{\mathrm{III}} E_{\mathrm{III}}$ showing increased post-translational modification product of apparent higher molecular weight. 


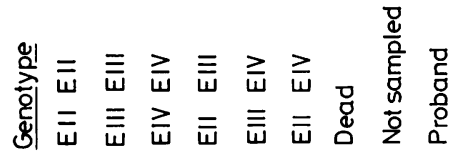

$\square \square \square \square$ שே
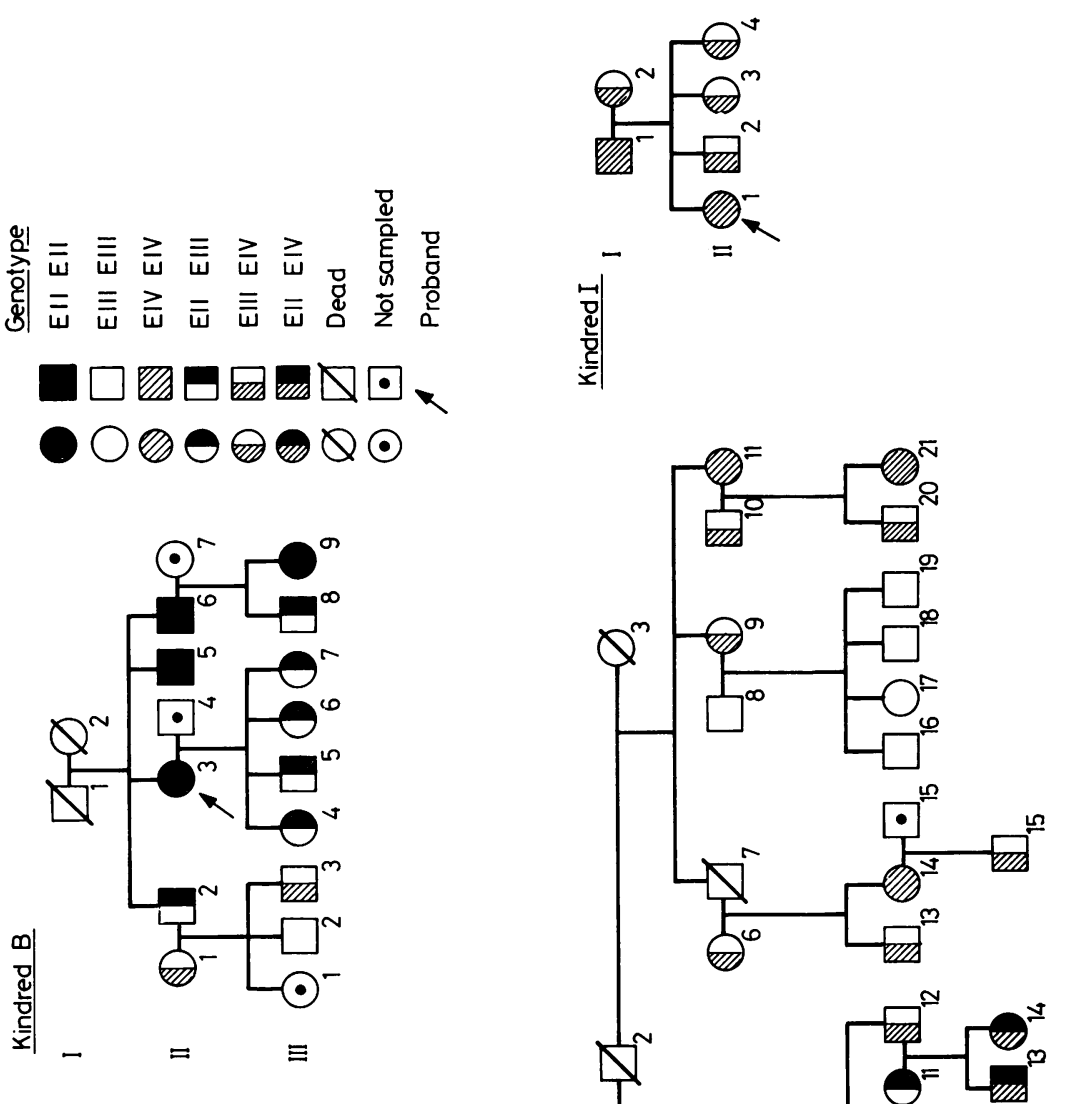

בे

○

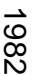
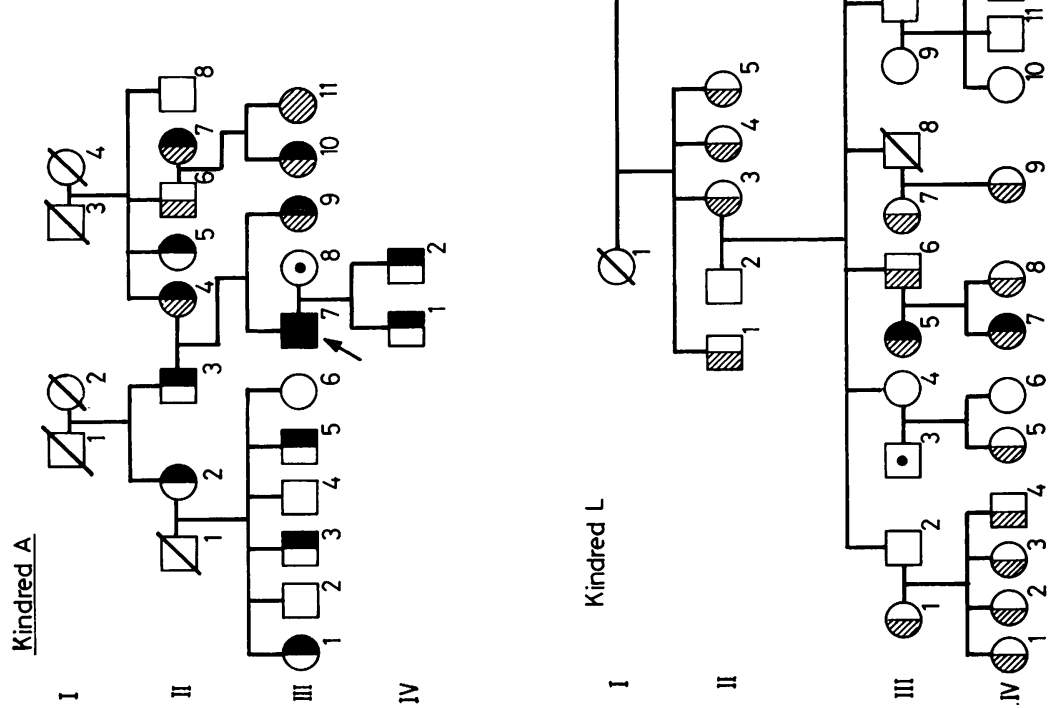
second differs in molecular weight as well as charge. Progressively in the anodic direction there is a tendency for an increase in apparent molecular weight as relative concentration of the isoprotein decreases.

Electrophoretograms (b), (d), and (f) in fig 2 represent examples where two major bands are present and these appear to be of the same or similar molecular weight, although they differ in charge. The minor, more anodic, isoproteins again show increasing apparent molecular weight with decreasing concentrations.

When equal concentrations of VLDL from different homozygotes are mixed, the resulting two dimensional pattern of the 'artificial heterozygote' closely resembles that of the corresponding natural heterozygote.

The simplest interpretation of the two dimensional evidence is that we are dealing with a single locus and three alleles which determine, when homozygous, the presence of a single major band in one or other of three positions, while the three alternative types with two major bands correspond to the three kinds of heterozygotes.

\section{NOMENCLATURE}

Originally the isoelectric bands were denoted by the letter ' $E$ ' followed by a Roman numeral to indicate the relative band position, for example, $\mathrm{E}_{\mathrm{I}}, \mathrm{E}_{\mathrm{II}}, \mathrm{E}_{\mathrm{III}}$, and $\mathrm{E}_{\mathrm{IV}}$, progressively in the direction of the cathode. More recently the Roman has been replaced with an Arabic numeral. In our opinion the latter system should be retained to denote band position by isoelectric focusing, while the Roman numeral should be used to indicate the three alleles as defined by their major band position. The presence of a single major band at the isoelectric positions $E_{4}, E_{3}, E_{2}$ would correspond respectively to genotypes $\mathrm{E}_{\mathrm{IV}} \mathrm{E}_{\mathrm{IV}}, \mathrm{E}_{\mathrm{III}} \mathrm{E}_{\mathrm{III}}$, and $\mathrm{E}_{\mathrm{II}} \mathrm{E}_{\mathrm{II}}$. The simul- taneous presence of major bands at two positions, for example, $E_{4}$ and $E_{3}$, or $E_{4}$ and $E_{2}$, or $E_{3}$ and $E_{2}$ would correspond respectively to the genotypes $\mathrm{E}_{I V} \mathrm{E}_{\mathrm{III}}, \mathrm{E}_{\mathrm{IV}} \mathrm{E}_{\mathrm{II}}$, and $\mathrm{E}_{\mathrm{III}} \mathrm{E}_{\mathrm{II}}$.

FAMILY STUDIES

Only four of the 12 families studied are described in fig 3 which includes the method of identifying genotypes. The families were chosen either on the evidence of type III hyperlipoproteinaemia in the probands (kindreds $\mathbf{A}$ and $\mathbf{B}$ ), earlier evidence of hypobetalipoproteinaemia among first degree relatives, or band pattern differences which looked particularly informative (kindreds I and $\mathbf{L}$ ).

All the family groups studied display inheritance consistent with the one locus three allele model. In particular, all the progeny of an $\mathrm{E}_{\mathrm{II}} \mathrm{E}_{\mathrm{II}}$ parent apparently carry an $\mathrm{E}_{\mathrm{II}}$ allele, as in kindreds $\mathrm{A}$ and $B$ in fig 3. Also, both parents of an $E_{I I} E_{I I}$ subject carry the $\mathrm{E}_{\mathrm{II}}$ allele, as in kindred $\mathrm{A}$. Corresponding relationships hold for the $E_{I V}$ allele (kindreds $A, I$, and $L$ ). The genotypes of $E_{I V} E_{I I}$ persons have always proved compatible with that of their parents. In crosses between persons of $\mathrm{E}_{\mathrm{III}} \mathrm{E}_{\mathrm{III}}$ genotypes no allele other than $\mathrm{E}_{\mathrm{III}}$ has been noted in their progeny.

In the survey of genotypes isoelectric focusing alone can be used for an accurate identification in about $80 \%$ of the cases; when there is doubt the matter is resolved by two dimensional electrophoresis. The nature of the problem with unidimensional isoelectric focusing is revealed in the distribution of the ratios of the concentration between pairs of apparent 'major' bands. These might arise either from the presence of dissimilar alleles or from a homozygous condition, controlling a single major band, together with a high level of posttranslational modification.

Fig 4 illustrates the position in terms of the ratio of band intensity at $E_{3}$ to band intensity at the

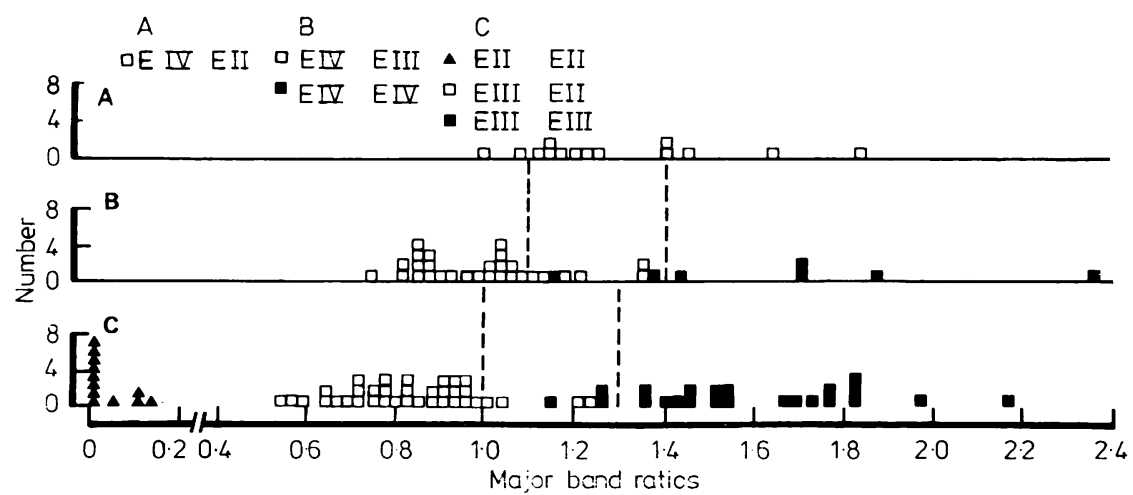

FIG 4 Distribution of ratios of band intensity $\left(E_{3} / E_{2}\right)$ in different genstypes. $(A) E_{\mathrm{IV}} E_{\mathrm{II}} ;(B) E_{\mathrm{IV}} E_{\mathrm{III}} ;(C) E_{\mathrm{III}} E_{\mathrm{II}}$. 
$E_{2}$ position in persons who lack an $E_{4}$ band and which therefore includes genotypes $\mathrm{E}_{\mathrm{II}} \mathrm{E}_{\mathrm{II}}, \mathrm{E}_{\mathrm{II}} \mathrm{E}_{\mathrm{III}}$, and $\mathrm{E}_{\mathrm{III}} \mathrm{E}_{\mathrm{III}}$. The distribution of ratios shows an area of overlap between 1.0 and $1 \cdot 3$. Similarly there is a low risk of confusion between $\mathrm{E}_{I I I} \mathrm{E}_{I V}$ and $E_{I V} E_{I V}$ from the linear scan in the ratio of $E_{4}$ to $E_{3}$.

Such variation in major bands may arise from several causes. Firstly, in some heterozygotes there may be variation in the two major bands, with only minor variation in the post-translational products. Fig 2(f) presents an example of this kind. Secondly, the relative concentration of post-translational products of apparently higher molecular weight differs in some subjects. Fig 2(c) and (i) show two dimensional Apo-E patterns from different subjects with the $\mathrm{E}_{\mathrm{III}} \mathrm{E}_{\mathrm{III}}$ genotype. In the latter case the relative concentration of the higher molecular weight peptide is enhanced. Thirdly, there may be variation in relative concentrations of post-translational products of apparently the same molecular weight. Fig 2(g) and (h) show Apo-E patterns from a single person sampled on different dates (see below). We can find no evidence that such differences are derived from alterations in procedure since repeated electrophoresis on the same VLDL sample, processed at different times, led to almost identical patterns while, in most cases, Apo-E patterns in samples taken from the same person at different times gave rise to very similar patterns. Also, Apo-E separated from different lipoprotein fractions, IDL (intermediate density lipoprotein) and LDL, gave rise to patterns very similar to those produced by Apo-E of VLDL origin for a given subject.

The causes of such variation are obscure. However, incubating VLDL with neuraminidase for 2 hours simplifies the Apo-E pattern by shifting the isoproteins of higher molecular weight to the same isoelectric point as the major band in homozygotes, although the first post-translational isoprotein of the same apparent molecular weight remains unaffected by this treatment. Zannis and Breslow ${ }^{11}$ have provided similar evidence on lyophylysed VLDL. Thus, an apparent molecular weight difference accompanied by charge difference may result from addition to the major peptide of one or more carbohydrate chains containing a terminal sialic acid residue.

However, neuraminidase treatment does not appear to alter the relative concentration of the particular isoprotein with the same apparent molecular weight as that of the single major band. It is possible that it may originate via an alternative post-translational pathway. Zannis and Breslow ${ }^{11}$ failed to affect it with either neuraminidase or acid or alkaline phosphatase and so it is unlikely that carbohydrate change or phosphorylation are respon-
TABLE Post-translational modification. Angular transformed proportion of Apo-E protein (except $E_{I}$ ) owing to post-translational modification in genotype $E_{I I I} E_{I I I}$.

\begin{tabular}{llc}
\hline Category & No & $\begin{array}{c}\text { Genotype } E_{\mathrm{III}} E_{\mathrm{III}} \\
(\text { Mean } \pm S E)\end{array}$ \\
\hline Controls & 80 & $43 \cdot 37 \pm 0 \cdot 44$ \\
Renal failure-dialysis & 20 & $43 \cdot 60 \pm 0 \cdot 74$ \\
Renal failure-transplant & 10 & $44 \cdot 05 \pm 1 \cdot 03$ \\
Survivors of infarction & 47 & $43 \cdot 88 \pm 0 \cdot 50$ \\
Hyperthyroid & 5 & $51 \cdot 20 \pm 2 \cdot 51$ \\
(1) Deviation from controls & & $7 \cdot 8^{*}$ \\
(2) Pre-treatment-post- & & $7 \cdot 0^{*}$ \\
\hline
\end{tabular}

*indicates significance of deviation from control average (1) or from nil difference (2)

sible for the origin of this particular isoprotein. On the other hand we have noted a change in its relative concentration in hyperthyroid patients. Fig 1(g) and $(\mathrm{h})$ show isoelectric patterns from a hyperthyroid patient in the untreated and euthyroid state. The results of two dimensional electrophoresis in the same samples can be seen in fig $2(\mathrm{~g})$ and $(\mathrm{h})$, showing that the increase resides in the isoprotein with the same apparent molecular weight as the major band.

Further evidence of post-translational modification in relation to thyroid activity is presented in the table by expressing the sum of the densitometric reading for the post-translational bands $E_{0}$ and $E_{2}$ as a proportion of the total $\left(E_{0}+E_{2}+E_{3}\right) . E_{1}$ is excluded because two dimensional electrophoresis reveals that this band includes an additional protein of different molecular weight and uncertain affinity. The percentages have been angular transformed for statistical comparison and are expressed in the transformed state in the table.

Several categories of patient who tend to have abnormal lipoprotein profiles, for example, survivors of myocardial infarction, renal failure, and transplant patients, do not significantly differ from the healthy controls in the post-translational index. The sexes are combined since there was no evidence of a sex difference. However, the hyperthyroid patients studied have significantly higher ratios than the controls $(p<0 \cdot 001)$. We also have records of modification of activity in hyperthyroid patients before and after treatment designed to return them to normality. In all cases there was a decline in the index values after treatment and the mean difference is highly significant.

\section{Discussion}

The study of Apo-E isoproteins by isoelectric focusing and two dimensional electrophoresis has shown that we can account for the complex patterns by a three allele single locus polymorphism. Each 
of the three alleles determines the presence of a major protein with a characteristic isoelectric point together with a number of minor proteins, some of which differ in charge, while others differ in both charge and apparent molecular weight. After isoelectric focusing the minor isoproteins are located at positions corresponding to 1,2 , or 3 units of charge difference from their reference major protein. This model is in complete agreement with the hypothesis of Zannis and Breslow ${ }^{11}$ and Zannis et al ${ }^{12}$ and contrary to the model proposed by Utermann et al. 5

Family studies show that alleles are inherited in a simple Mendelian fashion with co-dominant expression. We have not encountered exceptions to this model. Although the $\mathrm{E}_{\mathrm{II}} \mathrm{E}_{\mathrm{II}}$ genotype appears essential for manifestation of type III hyperlipoproteinaemia, the presence of subjects of this genotype but with normal lipoprotein profiles in several kindreds suggests that additional factors, either genetic or environmental, are required for development of type III hyperlipidaemia, as Utermann et al ${ }^{5}$ have suggested. In kindreds containing subjects with below average LDL serum concentrations, the $E_{\text {II }}$ frequency was high, in agreement with the finding of a cholesterol lowering effect of this allele in first degree relatives of type III patients. So far alleles $\mathrm{E}_{\mathrm{III}}$ and $\mathrm{E}_{\mathrm{IV}}$ have not been associated with any of the recognised hyperlipidaemia conditions.

The variation in presumably post-translational products involves changes in either charge alone or both charge and molecular weight. With respect to the former, neuraminidase does not affect the relative concentration of the isoprotein immediately anodic to the main band. Phosphorylation cannot yet be entirely excluded until a wider range of phosphatase specificity has been tested.

Where both charge and molecular weight changes occur, treatment with neuraminidase leads to a simplification of the Apo-E pattern, with a trailing band at the major isoprotein position, suggesting the initial presence of carbohydrate side chain(s) carrying terminal sialic acid residue(s).

The association between post-translational activity and departure from the normal thyroid status may provide a useful clue to the origins of post-translational modification generally. Hyperthyroidism apparently increases the number as well as the concentration of isoproteins of apparently similar molecular weight. It is not clear from the two dimensional pictures whether such modification in these subjects affects carbohydrate-containing proteins or involves further modification of the major proteins. If the latter is so, a particular amino-acid in the major peptide may have been modified at a number of sites in the molecule.

The Apo-E polymorphism presents us with a useful tool for study of serum lipoprotein metabolism, while the genetically controlled alterations of lipoprotein profiles may have implications for coronary risk. Also, the origin of the gene-gene or gene-environment interaction which involves the homozygous state of $\mathrm{E}_{\mathrm{II}}$, and which may give rise to highly abnormal lipoprotein profiles, poses a challenge which may also apply to the heterozygotes. Work is in progress to shed light on these aspects.

\section{References}

1 Shore B, Shore V. Heterogeneity of human plasma very low density lipoproteins. Separation of species differing in protein components. Biochemistry 1973;12:502-7.

2 Shelburne FA, Quarfordt SH. A new apoprotein of human plasma very low density lipoproteins. J Biol Chem 1974;249:1428-33.

3 Havel RJ, Kane JP. Primary dysbetalipoproteinaemia type III: deficiency of a specific apolipoprotein (Apo-E III) in the very low density lipoproteins. Proc Natl Acad Sci USA 1973;70:2015-19.

4 Utermann G, Jaeschke M, Menzel J. Familial hyperlipoproteinaemia type III : deficiency of a specific apolipoprotein (Apo-E-III) in the very low density lipoproteins. Febs Letters 1975;56:352-5.

5 Utermann G, Vogelberg KH, Steinmetz A, et al. Polymorphism of apolipoprotein E. II. Genetics of hyperlipoprotein type III. Clin Genet 1979;15:37-62.

6 Zannis VI, Breslow JL. Characterization of a unique human apolipoprotein $\mathrm{E}$ variant associated with type III hypolipoproteinaemia. J Biol Chem 1980;255:1759-62.

7 Robertson FW, Cumming AM. Genetic and environmental variation in serum lipoproteins in relation to coronary heart disease. J Med Genet 1979;16:85-100.

8 Warnick GR, Mayfield C, Albers JJ, Hazzard WR. Gel isoelectric focusing method for specific diagnosis of familial hyperlipoproteinaemia type 3. Clin Genet 1979; 25:279-84.

9 Reisner AH, Nemes P, Bucholtz C. The use of Coomassie Brilliant Blue $G 250$ perchloric acid solution for staining in electrophoresis and isoelectric focusing in polyacrylamide gels. Anal Biochem 1975;64:509-16.

10 O'Farrell PH. High resolution two-dimensional electrophoresis of proteins. J Biol Chem 1975;250:4007-21.

11 Zannis VI, Breslow JL. Human very low density lipoprotein apolipoprotein $\mathrm{E}$ isoprotein polymorphism is explained by genetic variation and post-translational modification. Biochemistry $1981 ; 20: 1033-41$.

12 Zannis VI, Just PW, Breslow JL. Human apolipoprotein $\mathrm{E}$ isoprotein subclasses are genetically determined. $\mathrm{Am} \mathrm{J}$ Hum Genet $1981 ; 33: 11-24$.

Requests for reprints to Professor F W Robertson, Department of Genetics, University of Aberdeen, 2 Tillydrone Avenue, Aberdeen AB9 2TN. 